\title{
Body Lateropulsion With Involvement of the Medial Lemniscus Due to a Middle Pontine Tegmentum Infarction
}

\author{
Hiromasa Tsuda ${ }^{a}$,, Tomoko Fujita ${ }^{a}$
}

\begin{abstract}
A 53-year-old man with essential hypertension abruptly developed rightward body lateropulsion (BL), and mild dysesthesia in the right upper extremity. No other neurologic abnormalities were observed. Cranial magnetic resonance imaging demonstrated a small infarct lesion in the middle pontine tegmentum on the left side. Under antiplatelet agent, the patient became asymptomatic within 7 days. BL is defined as irresistible falling to one side without motor weakness. Damage to the ascending graviceptive pathway (GP) may cause BL. GP from the vestibular nuclei to the Cajal interstitial nucleus crosses the midline in the caudal pons. Based on previous reports, the location of GP was speculated in the rostral pons. However, its precise location remains unreported in the middle pons. This is a second reported case of $\mathrm{BL}$ due to a middle pontine tegmentum infarction. In addition, findings of neurologic and magnetic resonance imaging in our patient suggest that GP may run in the dorsal region to the medial lemniscus in the middle pons.
\end{abstract}

Keywords: Ascending graviceptive pathway; Magnetic resonance imaging; Pons

\section{Introduction}

Body lateropulsion (BL) is defined as irresistible falling to one side without motor weakness $[1,2]$. BL is caused by damage to the ascending graviceptive pathway (GP). GP

Manuscript accepted for publication December 9, 2013

\footnotetext{
${ }^{a}$ Department of Neurology, Tokyo Metropolitan Health and Medical Corporation Toshima Hospital, Japan

${ }^{\mathrm{b}}$ Corresponding author: Hiromasa Tsuda, Department of Neurology, Tokyo Metropolitan Health and Medical Corporation Toshima Hospital, 33-1, Sakaecho, Itabashi-ku, 173-0015 Tokyo, Japan. Email: hiromasa_tsuda@tokyo-hmt.jp
}

doi: http://dx.doi.org/10.4021/jmc1612w from the vestibular nuclei to the Cajal interstitial nucleus crosses the midline in the caudal pons [1]. However, there was only one reported case of BL due to a middle pontine tegmentum infarction [1]. Therefore, precise location of GP remains uncertain in the middle pons. Here, we describe a case of BL with dysesthesia on the upper extremity due to a middle pontine tegmentum infarction. This case is very useful for determinating the location of GP in the middle pons.

\section{Case Report}

A 53-year-old man with essential hypertension abruptly presented with rightward $\mathrm{BL}$, and mild dysesthesia on the right upper extremity in December 2012. There were no other neurologic abnormalities. Laboratory examination was within normal ranges. Chest X-ray finding was normal. Electrocardiogram demonstrated no abnormalities. Diffusionweighted magnetic resonance (MR) imaging demonstrated a small infarct lesion in the rostral pontine tegmentum on the left side (Fig. 1). Cranial MR angiography finding was normal. Intravenous sodium ozagrel $160 \mathrm{mg} /$ day was initiated. Thereafter, the patient became gradually asymptomatic within 7 days.

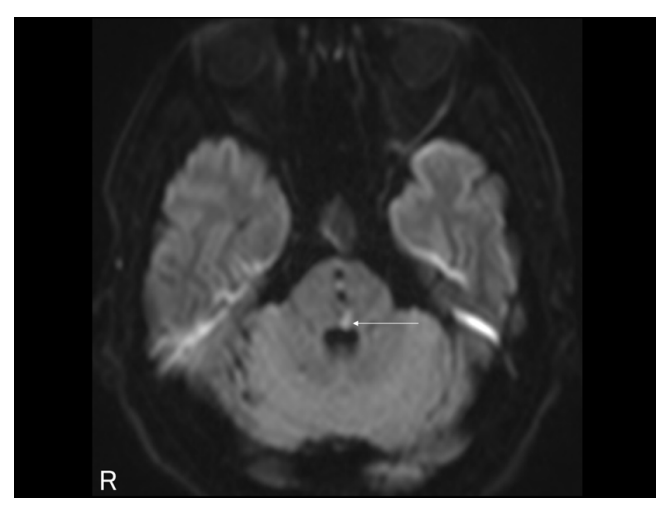

Figure 1. Cranial diffusion-weighted magnetic resonance imaging. A small infarction was demonstrated in the middle pontine tegmentum on the left side (arrow). 


\section{Discussion}

Yi et al [1] noted that GP from the vestibular nuclei to the Cajal interstitial nucleus crosses the midline in the caudal pons, just above the level of the vestibular nuclei [1]. Moreover, because unilateral internuclear ophthalmoplegia was observed in four cases of a rostral pontine tegmentum infarction, the authors speculated that GP might run in the vicinity of the medial longitudinal fasciculus [1]. Tsuda et al [2] noted a case of BL with hypalgesia and thermophypoesthesia in the territory of all divisions of the trigeminal nerve caused by a rostral pontine tegmentum infarction, and stated that GP might run in the vicinity of the ventral trigeminothalamic tract in the rostral pons. On the other hand, in the middle pons, the precise location of GP remains uncertain. Yi et al [1] reported a first case of BL due to a middle pontine tegmentum infarction, and the direction of BL was to the side opposite the lesion. However, no other neurologic abnormalities were detected. In our present patient, in addition to rightward $\mathrm{BL}$, mild dysesthesia on the upper extremity was induced by small infarct lesion in the left middle pontine tegmentum. This is a second reported case of BL due to a middle pontine tegmentum infarction. Based on cranial
MR imaging finding, mild dysesthesia on the upper extremity might result from partial involvement of the left medial lemniscus. In conclusion, we emphasize that GP may run in the dorsal region to the medial lemniscus in the middle rostral pons.

\section{Grant Support}

None.

\section{References}

1. Yi HA, Kim HA, Lee H, Baloh RW. Body lateropulsion as an isolated or predominant symptom of a pontine infarction. J Neurol Neurosurg Psychiatry. 2007;78(4):372-374.

2. Tsuda H, Koh S, Tanaka K. Body lateropulsion with hypalgesia and thermophypoesthesia in the territory of all divisions of the trigeminal nerve caused by a pontine infarction. Jpn J Stroke. 2013;35(3):213-215. 